Reprod. Nutr. Dévelop., 1988, 28 Suppl. n 1, 95-96

\title{
Traitement des coques de tournesol par vapocraquage : influence sur la composition et la dégradation in sacco des polyosides pariétaux
}

\author{
J. L. BARRY, M. VIGNON $\left({ }^{*}\right)$, G. EXCOFFIER $\left({ }^{*}\right)$, S. GUENEAU, Agnès DAVID
}

Laboratoire de Technologie Appliquée à la Nutrition,

I.N.R.A., B.P. 527, 44026 Nantes Cedex 03, France.

(') CERMAV, C.N.R.S., B.P. 68, 38402 St-Martin-d'Hères Cedex.

Summary. Steam-explosion of sunflower hulls leads to 12 to $20 \%$ dry matter losses mainly consisting of non cellulosic cell-wall polysaccharides. Dry matter ruminal disappearance is inhanced by 45 to $73 \%$, the best improvement beeing for xylose. Drying after treatment may have limited the enhancement of cellulose degradation.

Le traitement préalable par vapocraquage des coques de tournesol (téguments de la graine) permet d'obtenir par action enzymatique ultérieure une saccharification importante de la cellulose de ce substrat (Dekker et Wallis, 1983). Le présent travail a pour objet d'étudier la possibilité d'employer le vapocraquage pour améliorer la dégradation dans le rumen des coques de tournesol, particulièrement peu fermentescibles à l'état natif.

Matériel et méthodes. Deux lots de coques de tournesol, broyées (lot 1) ou entières (lot 2), ont été traités par vapocraquage (vapeur à 35 bars pendant $1 \mathrm{~min}$, puis explosion par détente), récupérés quantitativement et lyophilisés. La dégradabilité des coques non traitées et traitées a été mesurée in sacco (4 chèvres, 4 sachets par animal, $48 \mathrm{~h}$ ). La composition en oses neutres pariétaux cellulosiques (glucose) et hémicellulosiques (rhamnose, arabinose, xylose, mannose et galactose) a été déterminée sur les produits traités et non traités (produit total et fraction insoluble dans l'éthanol $80^{\circ}$ ) et sur les résidus de fermentation, selon la technique de Hoebler et al. (1989), par hydrolyse sulfurique puis analyse chromatographique.

Résultats et discussion. La cellulose, non affectée au cours du traitement (Targonsky, 1985), permet d'estimer les pertes de matière sèche (MS) provoquées par le traitement à respectivement 12 et $20 \%$ pour les lots 1 et 2 . Le traitement diminue les teneurs en hémicelluloses respectivement de 58 et $75 \%$ pour les lots 1 et 2 (tabl. 1). Ces pertes représentent respectivement 10 et $14 \%$ de la MS initiale et 83 et $70 \%$ des pertes totales de MS. Une fraction des hémicelluloses résiduelles est constituée de molécules de faible degré de polymérisation $\left(<12\right.$ ) solubles dans l'éthanol $80^{\circ}$. Le vapocraquage augmente la disparition in sacco de la MS des coques (respectivement de 45 et de $73 \%$ pour les lots 1 et 2) (tabl. 2). La dégradation des oses majeurs, notamment du xylose, est augmentée, même en l'absence de broyage avant traitement. Cette améliora- 
tion est plus importante pour les hémicelluloses que pour la cellulose, dont la disparition est nettement inférieure aux taux de dégradabilité enzymatique mesurés antérieurement (Dekker et Wallis, 1983). Le séchage après traitement, provoquant un affaissement dans la structure des fibres de cellulose (Buléon et Bertrand, 1982), pourrait expliquer la relative inefficacité du traitement à ce niveau. L'acidité des produits traités $(\mathrm{pH}=4,5)$ permet d'envisager leur conservation sous forme humide et donc une meilleure efficacité du traitement.

TABL. 1. - Influence du vapocraquage sur la teneur en oses et polyosides pariétaux (\% MS) et la fraction soluble dans l'éthanol $80^{\circ}$ (\% sol.).

\begin{tabular}{|c|c|c|c|c|c|c|c|c|}
\hline & \multicolumn{4}{|c|}{ Coques broyées } & \multicolumn{4}{|c|}{ Coques entières } \\
\hline & \multicolumn{2}{|c|}{ Non traitées } & \multicolumn{2}{|c|}{ Traitées } & \multicolumn{2}{|c|}{ Non traitées } & \multicolumn{2}{|c|}{ Traitées } \\
\hline & $\% \mathrm{MS}$ & $\%$ sol. & $\% \mathrm{MS}$ & $\%$ sol. & $\% \mathrm{MS}$ & $\%$ sol. & $\% \mathrm{MS}$ & $\%$ sol. \\
\hline Rhamnose $\left({ }^{1}\right)$ & 0,7 & 0 & 0 & - & 0,8 & 0 & 0,4 & 100 \\
\hline Arabinose & 2,3 & 0 & 0,5 & 100 & 2,4 & 0 & 0 & - \\
\hline Xylose & 12,4 & 0 & 6,1 & 23 & 12,8 & 0 & 3,6 & 28 \\
\hline Mannose & 1,1 & 0 & 0,7 & 14 & 1,0 & 0 & 0,7 & 14 \\
\hline Galactose & 1,0 & 0 & 0,5 & 100 & 1,0 & 0 & 0 & - \\
\hline Hémicelluloses $\left({ }^{2}\right)$ & 17,4 & 0 & 5,3 & 12 & 17,9 & 0 & 4,5 & 29 \\
\hline Cellulose $\left({ }^{3}\right)$ & 23,7 & 0 & 27,1 & 0 & 25,3 & 0 & 31,5 & 0 \\
\hline
\end{tabular}

TABL. 2. - Influence du vapocraquage sur la disparition dans le rumen (\%) de la MS, des oses et polyosides pariétaux (moyenne \pm erreur standard).

\begin{tabular}{|c|c|c|c|c|}
\hline & \multicolumn{2}{|c|}{ Coques broyées } & \multicolumn{2}{|c|}{ Coques entières } \\
\hline & Non traitées & Traitées & Non traitées & Traitées \\
\hline MS & $26,4 \pm 1,2$ & $38,0 \pm 1,5$ & $20,7 \pm 1,9$ & $35,9 \pm 1,2$ \\
\hline Rhamnose & $60,5 \pm 3,1$ & 二 & $89,5 \pm 10,6$ & $24,5 \pm 45,5$ \\
\hline Arabinose & $47,6 \pm 2,1$ & $91,4 \pm 8,6$ & $52,0 \pm 1,9$ & - \\
\hline Xylose & $13,7 \pm 3,2$ & $59,2 \pm 2,0$ & $3,3 \pm 2,4$ & $52,3 \pm 1,9$ \\
\hline Mannose & $73,3 \pm 1,1$ & $30,2 \pm 17,0$ & $49,1 \pm 5,0$ & $31,6 \pm 11,3$ \\
\hline Galactose & $53,1 \pm 1,4$ & $84,9 \pm 15,2$ & $46,2 \pm 4,5$ & 二 \\
\hline Hémicelluloses & $25,9 \pm 2,7$ & $57,0 \pm 3,0$ & $18,2 \pm 1,5$ & $45,6 \pm 2,7$ \\
\hline Cellulose & $23,0 \pm 2,9$ & $32,3 \pm 4,2$ & $21,8 \pm 2,5$ & $28,3 \pm 1,4$ \\
\hline
\end{tabular}

Buléon A., Bertrand D., 1982. J. appl. Biochem., 4, 218-227.

Dekker R. F. H., Wallis A. F. A., 1983. Biotechnol. Letters, 5, 311-316.

Hoebler C., Barry J. L., David A., Delort-Laval J., 1989. J. agric. Food Sci. (sous presse).

Targonsky Z., 1985. Acta Biotechnol., 5, 353-361. 